\title{
Pink hypopyon: a sign of Serratia marcescens endophthalmitis
}

\author{
Selwa A F Al Hazzaa, Khalid F Tabbara, J Allan Gammon
}

\begin{abstract}
Endogenous bacterial endophthalmitis in infants is uncommon. We recently examined and treated an infant who presented with pink hypopyon which followed a Serratia marcescens septicaemia. Culture of the aspirate from the anterior chamber showed no red blood cells, and grew Serratia marcescens, which was also isolated from the tip of the child's umbilical artery catheter. The presence of a pink hypopyon in the absence of hyphaema may suggest the diagnosis of Serratia marcescens endophthalmitis.
\end{abstract}

(Brf Ophthalmol 1992; 76: 764-765)

Serratia marcescens, a Gram negative bacillus once believed to be a harmless saprophyte may cause infection in humans. This bacterium can cause meningitis, endocarditis, otitis media, sepsis, and ocular infections such as conjunctivitis and keratitis. ${ }^{1-3}$ Endogenous bacterial endophthalmitis is uncommon but its occurrence in newborns has been reported. ${ }^{2}$ We report a newborn infant who developed a pink hypopyon caused by Serratia marcescens.

\section{Case report}

After an uneventful pregnancy, a healthy 18year-old primigravida had an uncomplicated labour and delivered a $2.65 \mathrm{~kg}$ full term infant. The neonate required resuscitation after delivery. Apgar scores were 1 at 5 minutes, 3 at 6 minutes, and 5 at 10 minutes. During the first few hours of life, the baby developed convulsions and was treated with $60 \%$ oxygen and phenobarbitone. Three days later the infant developed clinical signs of septicaemia and disseminated intravascular coagulopathy. Serratia marcescens was isolated from blood cultures and from the infant's ear, nose, and throat. $S$ marcescens was also cultured from the tip of the child's umbilical artery catheter.

Ophthalmic examination disclosed a normal right eye. The left cornea was hazy centrally. A fibrinous exudate was seen in the anterior chamber occluding the pupil and precluding visualisation of the fundus. A pink hypopyon occupied one third of the anterior chamber. Ultrasound evaluation of the posterior segment revealed dense, dispersed vitreous opacities and retinochoroidal thickening consistent with endophthalmitis. No intraocular calcification was noted.

An anterior chamber paracentesis was performed. Gram stain of the aqueous specimen revealed Gram negative bacilli and polymorphonuclear cells. Haematoxylin and eosin stain of the fibrinous exudate showed polymorpho- nuclear leucocytes but not red blood cells. Serratia marcescens was isolated from the aqueous. Vitreous was aspirated for microscopy and culture. A 25 gauge needle on a tuberculin syringe was inserted through the pars plana at the 9 o'clock position and was advanced less than $1 \mathrm{~cm}$ to reach the mid vitreous. Liquefied vitreous $(0.3 \mathrm{ml})$ was aspirated, and through the same site gentamicin $100 \mu \mathrm{g}$ and cephazolin $100 \mu \mathrm{g}$ were injected intravitreally. Culture of vitreous aspirate revealed no growth. The view of the fundus was not possible because of the hazy media.

Antibiotic sensitivity studies showed the organism to be susceptible to amikacin, chloramphenicol, carbenicillin, gentamicin, minocycline, and trimethoprim-sulphamethoxazole, and resistant to ampicillin, cephalosporins, erythromycin, polymyxin B, and tetracycline. Radiological examination of the extremities confirmed the presence of periosteal reaction suggestive of osteomyelitis. The patient responded to parenteral gentamicin therapy. The left eye showed progressive healing of the inflammatory reaction. Three months later the left eye started showing evidence of phthisis.

\section{Discussion}

Serratia marcescens was first described by Bizio in 1823. ${ }^{4}$ Subsequently, the bacterium became known as Bacterium prodigiosum because it produces a red pigment (prodigiosin). Its red pigment has been a useful marker in the study of bacteraemia after dental extractions ${ }^{5}$ and bacteriuria after urinary tract procedures. ${ }^{6} S$ marcescens endophthalmitis has rarely been reported. A review of 72 cases of metastatic endophthalmitis in the past 10 years disclosed only three cases of $S$ marcescens, all in the adult age group..$^{7-9}$

Endophthalmitis in infants has received little attention. Three cases of bacterial endophthalmitis reported in the neonatal age group were reported to have been caused by Streptococcus pneumoniae, Streptococcus algalactiae, and group B Streptococcus. ${ }^{10-12}$

Serratia marcescens ocular infections have been described as purulent conjunctivitis in six premature infants. ${ }^{13}$ In 1971 Bigger et al reported two cases of severe purulent endophthalmitis owing to $S$ marcescens in adults. ${ }^{14} \mathrm{We}$ assume that our patient had metastatic endophthalmitis following septicaemia acquired by umbilical artery catheter infection.

Because of the potential severity of ocular involvement by this organism, a characteristic presentation of pink hypopyon may suggest $S$ marcescens and should prompt the ophthalmologist to look for laboratory confirmation 
and institute immediate management with appropriate antibiotics such as gentamicin.

Endogenous metastatic endophthalmitis in infants is rare. Vitrectomy and intraocular antibiotic injections may be indicated in such patients but are technically demanding if the view is obstructed, and may carry considerable risks.

1 Gammon JA, Schwab I, Joseph P. Gentamicin-resistant Serratia marcescens endophthalmitis. Arch Ophthalmol 1980 98: 1221-3.

2 Lazacheck GW, Boyle GL, Schwartz AL, Leopold IH. Serratia marcescens, an ocular pathogen. Arch Ophthalmol 1971; 85: 599-603

3 Davis JT, Foltz E, Blackemore WS. Serratia marcescens: a pathogen of increasing clinical importance. $\mathcal{F} A M A$ 1970; 213: 2190-2.

4 Gaughran ER. From superstition to science. The history of a bacterium. Trans NY Acad Sci 1969; 31: 3-24.
5 McEntegart MG, Porterfield JS. Bacteremia following dental extractions. Lancet 1949; ii: 596-8.

6 Kass EH, Schneiderman LJ. Entry of bacteria into the urinary tracts of patients with inlaying catheters. $N$ Engl $\mathcal{f} M e d$ tracts of patients

7 Greenwald MJ, Wohl LG, Sell CH. Metastatic bacterial endophthalmitis: a contemporary reappraisal. Surv Ophthal-

8 Malnou F. Metastatic septique endoculaire per Serratia. Bull Soc Ophtalmol Fr 1981;81: 803-6.

9 Radda TM. Metastatic Serratia marcescens endophthalmitis. Ophthalmologica 1982;185: 65-8.

10 Greene GR, Carroll WL, Morozumi PA. Endophthalmitis associated with group-B streptococcal meningitis in an infant. Am $\mathcal{F}$ Dis Child 1979; 133: 752.

11 Weintraub MI, Otto RN. Pneumococcal meningitis and endophthalmitis in a newborn. $\mathcal{F} A M A 1972 ; 219$ : 1763-4.

12 Berger B. Endophthalmitis complicating neonatal group B streptococcal septicemia Am f Ophthalmol 1981;92:681-4.

13 Stenderup A, Faergeman O, Ingerslev M. Serratia marcescens inderup A, Faergeman $O$, Ingerslev M. Serratia marcescens infections in premature infants. Acta Pathol Microbiol Scand

14 Bigger JF, Meltzer G, Mandell A, Burde RM. Serratia marcescens endophthalmitis. Am $\mathcal{F}$ Ophthalmol 1971; 72: 